\title{
USO DE HERRAMIENTAS SOCIALES COMO APOYO DOCENTE
}

\author{
Carlos Meléndez Tamayo \\ Doctor en Innovación Educativa \\ Universidad Técnica de Ambato, Ecuador \\ cmelendez77@gmail.com \\ Víctor Hugo Abril Porras \\ PH. D in Agricultura \\ Universidad Técnica de Ambato, Ecuador \\ vhabril@gmail.com
}

\section{Cómo citar este artículo}

Meléndez, C. y Abril, V. (2013) Uso de herramientas sociales como apoyo docente. Espiral, Revista de Docencia e investigación. 3, (1) 9-14

\section{Resumen}

El presente trabajo muestra una metodología de uso y aplicaciones de diversas herramientas de la Web 2.0 integradas en el proceso de enseñanza-aprendizaje, constituidas como un recurso contemporáneo de apoyo alternativo para docentes. En ese sentido se combina y maneja varios instrumentos de la web social, con el propósito que los estudiantes se vinculen con los contenidos de las asignaturas, interactúen con sus pares y también con sus maestros fuera del aula, así como también, en su tiempo libre con actividades curriculares que permitirán mejorar, pero sin dejar de lado el rigor. El uso de las herramientas sociales de la web tiene como propósito generar conocimiento propio o significativo a través de un aprendizaje apoyado en las Tecnologías de la Información y Comunicación (TIC). El enfoque utilizado dentro de la metodología utilizada es mixto ya que se analizó una realidad educativa, así como también, se recopiló información de campo a través de encuestas aplicadas a seis docentes voluntarios y 110 estudiantes usuarios de la Facultad de Contabilidad y Auditoría de la Universidad Técnica de Ambato, ubicada en la provincia de Tungurahua - Ecuador.

Palabras clave: herramientas sociales, web 2.0, internet, innovación educativa, TIC.

\footnotetext{
Abstract

This job presents a methodology where the integration of various Web 2.0 tools in the teaching-learning process are used and applied, constituting as a contemporary means of alternative support for teachers. This means, combines and manages various social web tools, allowing students to get involved with the subject content, interacting with their peers, and with their teachers outside the classroom, especially in their free time in any rigid curricular activity. The usage of these tools in
}

the social web has the purpose to elicit self-knowledge through a meaningful learning supported by the Information Technology and Communication (ITC or TIC). The approach used in the methodology was qualitative and quantitative system which was analyzed in the educational system reality, as well as, a field data that was collected through surveys applied to six volunteer teachers and 110 enrolled students in the Accounting and Auditing Faculty of the Técnica de Ambato University, located in the province of Tungurahua - Ecuador.

Keywords: social tools, web 2.0, internet, educational innovation, $\mathrm{TIC}^{\prime} \mathrm{s}$

\section{INTRODUCCIÓN}

Una red social es una estructura agrupada de forma voluntaria que se puede representar en forma de uno o varios grafos en el cual los nodos representan individuos (a veces denominados actores) y las aristas, relaciones entre ellos. Las relaciones pueden ser de distinto tipo, como intercambios financieros, amistad, relaciones (hasta sexuales), o rutas aéreas. De Haro, J. (2010) sugiere también considerarla como el medio de interacción de distintas personas para intervenir en juegos en línea, chats o foros, entre otros.

Como ya se ha afirmado en otros estudios de nuestra autoría, la Web 2.0 es una red colaborativa, y por ende, cada vez más social. La característica fundamental es que los usuarios se 
convierten en colaboradores activos, publican información (individual o en conjunto), realizan cambios e intercambio en los datos, socialización, reutilización; interactúan con el sitio y con otros visitantes. Todo lo antes expuesto, se hace gracias a un sinnúmero de aplicaciones desarrolladas, de las cuales se pueden obtener múltiples ventajas, entre ellas, que los usuarios tengan facilidad de acceso a la información por consultas o investigaciones, facilidades de divulgación e interoperabilidad, con la única finalidad de estimular y aprovechar al máximo la inteligencia colectiva.

En lo referente a innovación educativa, se tiene que es de una naturaleza bastante compleja. Al respecto, Mayo \& Lank (2000) sugieren que la innovación no es sólo la aplicación de resultados de I+D (investigación y desarrollo a alto nivel), sino que también es el resultado de capacidades emprendedoras, estratégicas de decisión, organizativas e imaginativas. En el presente estudio se pretende analizar el sentido de la innovación dentro de la educación, con la incorporación de las TIC (tecnologías de la información y comunicación), y específicamente con uno de sus elementos como son las herramientas sociales.

En lo referente a estas tecnologías de la información y comunicación la Cumbre Mundial sobre la Sociedad de la Información (2003) en el numeral 9 menciona:

Somos conscientes que las TIC deben considerarse un medio, y no un fin en sí mismas. En condiciones favorables, estas tecnologías pueden ser un instrumento eficaz para acrecentar la productividad, generar crecimiento económico, crear empleos y fomentar la ocupabilidad, así como mejorar la calidad de la vida de todos. Pueden, además, promover el diálogo entre las personas, las naciones y las civilizaciones.

El aprendizaje significativo según la teoría constructivista está sustentado en la idea de in- teracción con los demás y a través de la conexión de conocimientos nuevos con aprendizaje previamente adquirido, Ballester, (2002). Se logra conectando la estrategia didáctica del profesorado con las ideas previas del alumnado, "construyendo" de manera sólida los conceptos, interconectando los unos con los otros en forma de red de conocimiento.

Con este marco conceptual base, se plantearon los objetivos del presente estudio. El objetivo general fue indagar el uso de las herramientas sociales de la web para la generación de un conocimiento significativo en la docencia universitaria mediante el apoyo de las TIC; y, los objetivos específicos fueron:

- Determinar el uso de las redes sociales para el establecimiento de la relación docente-estudiante fuera del aula.

- Establecer las aplicaciones de la web social para su ponderación en función de la utilidad que se les da en el proceso de enseñanza-aprendizaje.

- Identificar las herramientas de la web 2.0 desconocidas o subutilizadas dentro de la docencia para el planteamiento de futuras propuestas de aplicación.

\section{METODOLOGÍA}

El estudio tiene como punto de partida el enfoque cuali-cuantitativo, tomando como referencia lo indicado por Pérez (1994) sobre la investigación educativa como aquella "modalidad de investigación destinada a evaluar los programas educativos en condiciones de rigor de cara a la mejora de las personas a las que se aplica"(pág. 510). Las características principales de esta investigación son: practicidad y especificidad a realidades concretas, diversidad en los diseños de investigación ya que utiliza perspectivas desde el ámbito cuantitativo y cualitativo

La modalidad de la investigación en una primera instancia es de carácter bibliográfica- 
documental; entendiéndose como el propósito de conocer, comparar, ampliar, profundizar y deducir diferentes enfoques, teorías, conceptualizaciones y criterios de diversos autores sobre una cuestión determinada, basándose en documentos (fuentes secundarias). Posteriormente, se aplicó la modalidad de campo, de acuerdo a un estudio sistemático de los hechos en el lugar con la incorporación de las herramientas sociales de la Web 2.0 en que se producen los acontecimientos para tomar contacto en forma directa con la realidad, y obtener información de acuerdo con los objetivos del proyecto.

La técnica e instrumento aplicados, fueron la encuesta con un cuestionario estructurado de quince ítems de diferente tipo (por su naturaleza o contenido, por su función y por los grados de libertad), de las cuales ocho preguntas tuvieron relación directa con el estudio, a continuación se detallan las mismas:

1. ¿Conoce usted lo que es una red social?

2. ¿Utiliza redes sociales en el ámbito educativo?

3. ¿Cuál es la frecuencia de uso de estas redes como apoyo educativo?
4. ¿Qué tipo de red social utiliza frecuentemente?

5. ¿Conoce las aplicaciones de la web 2.0?

6. ¿Qué herramientas de la web 2.0 utiliza frecuentemente en educación?

7. ¿Valora la utilización de las herramientas de la web 2.0 en el proceso de enseñanza-aprendizaje?

8. ¿Conoce las aplicaciones de las herramientas de la web 2.0 enlistadas?

El universo participante en el estudio fue conformado por la comunidad universitaria de la Facultad de Contabilidad y Auditoría de la Universidad Técnica de Ambato, modalidad presencial. El lugar de estudio fue seleccionado en función del acceso que los autores tienen, al ser docentes investigadores de ese establecimiento. El tamaño de la población es igual al número de estudiantes por cursos de los profesores que se involucraron en el levantamiento de información de campo y se presentan en la Tabla 1. El tipo de muestreo utilizado para los docentes fue el no probabilístico decisional (factor decisional - integrantes de un área académica); $y$, para los estudiantes se aplicó el muestreo probabilístico regulado.

TABLA 1. Población de estudio y marco muestral

\begin{tabular}{|c|c|c|}
\hline COMUNIDAD UNIVERSITARIA & Población $-\mathbf{N}$ & Muestra $\boldsymbol{n}$ \\
\hline Profesores & 133 & 6 \\
\hline Estudiantes & 1756 & 110 \\
\hline TOTAL & 1889 & 116 \\
\hline
\end{tabular}

Fuente: Elaboración de los autores.

Es importante mencionar que se realizó un proceso de validación del cuestionario el mismo que fue desarrollado en tres fases, este proceso permitió contar con un instrumento de calidad, con el propósito de obtener información relevante y confiable:
1. Validación del cuestionario por parte de un experto, el mismo que permitió mejorar la estructura, redacción de las preguntas y relevancia para el procesamiento posterior. 
2. Rediseño del cuestionario, con base a las observaciones del experto.

3. Aplicación de una prueba piloto, en la que se pudo detectar dificultades por parte de los encuestados para contestar, ya sea por emplear un lenguaje extremadamente técnico y se aplicó aleatoriamente en un paralelo de la Facultad de Contabilidad y Auditoría conformado por 25 estudiantes.

\section{RESULTADOS}

En función del cumplimiento de los objetivos específicos se tiene:

1. El $88.25 \%$ de la comunidad universitaria utiliza las redes sociales fuera del aula como vínculo entre docentes y estudiantes con fines académicos, la aplicación Facebook es la de mayor empleo con un $88.01 \%$

FIGURA 1. Porcentaje de uso de redes sociales y aplicaciones
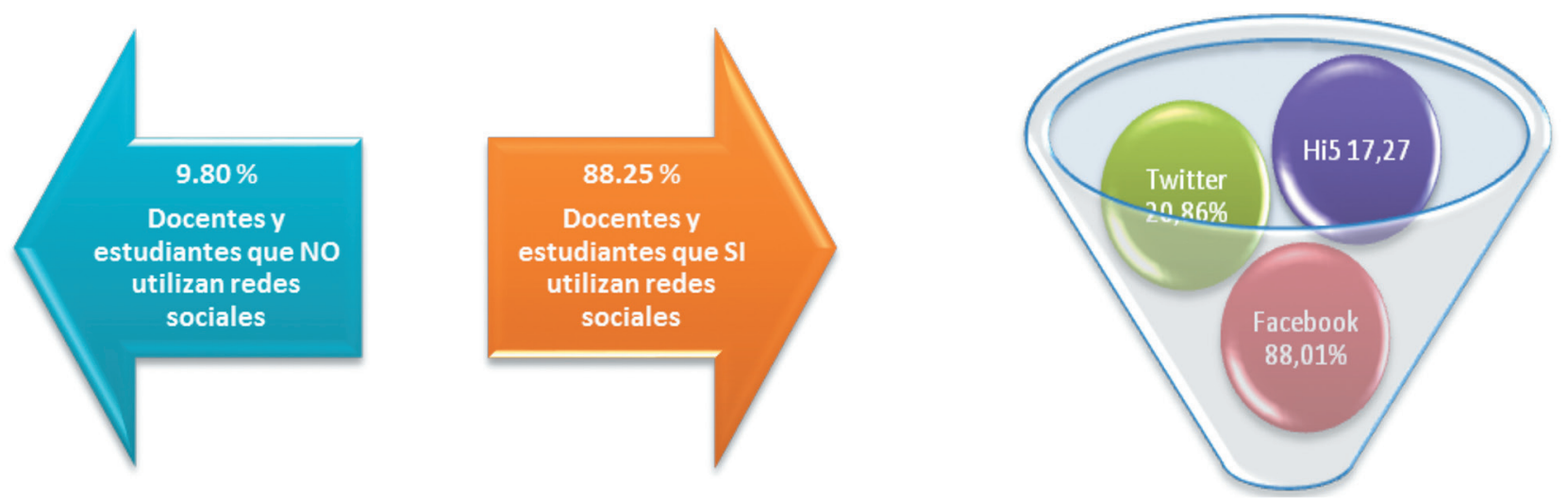

Fuente: Diseño de los autores

Las aplicaciones de la web social ponderadas que se establecieron en función de la utilidad que se les da en el proceso de enseñanza- aprendizaje fueron: Youtube, Chat, Blogs, Wiki, Skype y Slideshare en ese orden y con los resultados que se indican en la Figura 2.

FIGURA 2. Ponderación de aplicaciones de la web social

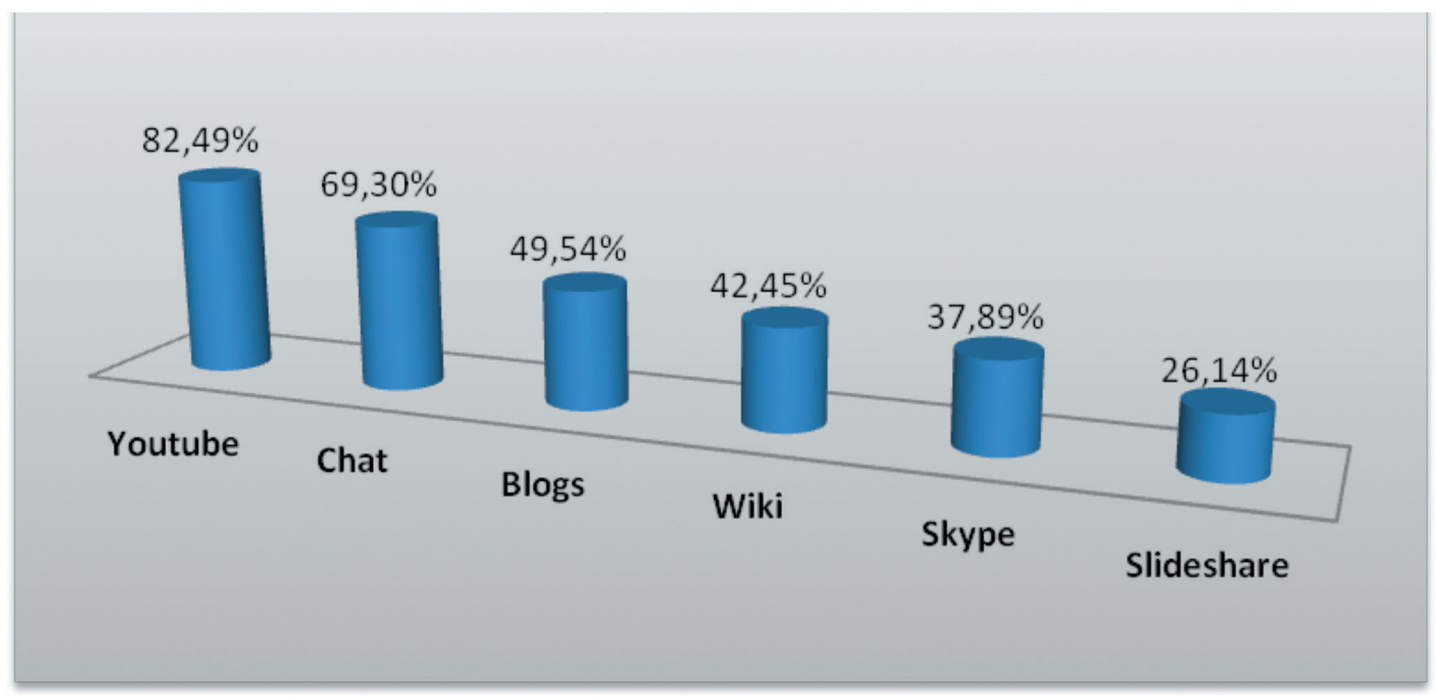

Fuente: Elaboración de los autores. 
2. Las herramientas de la web 2.0 que se identificaron como desconocidas o subutilizadas dentro de la docencia fueron: Misterwong, Podcast y Scribd.

3. Ante esta realidad es pertinente reconocer la contribución en la construcción del conocimiento que supone el uso de las herramientas sociales.

4. El aumento de usuarios con fines académicos de las herramientas sociales indica unas nuevas formas y códigos para la apropiación y el intercambio en la comunidad académica.

\section{CONCLUSIONES}

En función de los resultados obtenidos del cumplimiento de los objetivos específicos y del cuestionario aplicado se tiene:

1. La comunidad universitaria conoce y usa la red social como elemento fundamental de interacción virtual.

2. La comunidad universitaria utiliza las redes sociales en el ámbito educativo, a más de los personales y laborales.

3. La frecuencia de uso de las redes como apoyo educativo es alta, debido a que las usan casi a diario.

4. El Facebook es la red social de mayor uso, debido a su fácil acceso, rápida difusión, amplia aceptación y moda.

5. La comunidad universitaria conoce seis de las nueve aplicaciones de la web 2.0 bajo estudio, que tienen relación con el proceso de enseñanza-aprendizaje.

6. Las herramientas de la web 2.0 que son utilizadas frecuentemente en educación son las de multimedia educativa, intercambio e interacción de información online y comunicación virtual.
7. La comunidad universitaria valora la utilización de las herramientas de la web 2.0 en el proceso de enseñanza-aprendizaje, ya que le permite interactuar, innovar el proceso, acceder a información y facilitar la comunicación.

8. Lastimosamente, existen aplicaciones de las herramientas de la web 2.0 que no son utilizadas por su dificultad, limitaciones tecnológicas y desconocimiento de sus utilidades.

\section{RECOMENDACIONES}

1. Usar los resultados de este estudio como base para otro de mayor envergadura que cubra la totalidad de la institución de educación superior con sus diez facultades, a fin de conocer la realidad informática de la comunidad universitaria.

2. Fomentar el uso de las herramientas sociales como apoyo al proceso de enseñanza-aprendizaje.

3. Capacitar al cuerpo docente en el uso y aplicación de la web social como instrumento de soporte permanente que genere un aprendizaje significativo.

\section{BIBLIOGRAFÍA}

Abril P., Víctor Hugo (2003). Elaboración de proyectos de investigación científica. Programas de formación de cuarto nivel. Centro de estudios de posgrados de la Universidad Técnica de Ambato. Recuperado el 15 de enero de 2013, de http://vhabril.wikispaces. com/file/view/7UTA.\%20Metodolog\%C3\%ADa\%20 Investigaci\%C3\%B 3n\%20-\%20Abril\%20PhD. pdf/189098907/7UTA.\%20Metodolog\%C3\%ADa\%20 Investigaci\%C3\%B3n\%20-\%20Abril\%20PhD.pdf

Ballester, A. (2002). El aprendizaje significativo en la práctica. Como hacer el aprendizaje significativo en el aula. Palma.

De Haro, J. (2010). Redes sociales en educación (Colegio Amor de Dios. Barcelona). Recuperado el 12 de noviembre de 2010, de aplicaciones educativas de las redes sociales. Presented at the jornada DIM de 
otoño. Disponible en: http://www.slideshare.net/jjdeharo/aplicaciones-educativas-de-las-redessocialespresentation

Meléndez T., Carlos (2012). Plataformas virtuales como recurso para la enseñanza en la universidad: análisis, evaluación y propuesta de integración de Moodle con herramientas de la Web 2.0. Tesis Doctoral. Universidad Complutense de Madrid, Madrid.

Mayo, P., y Lank, J. (2000). Proceso educativo innovador. Argentina: Buenos Aires.
Cumbre Mundial sobre la Sociedad de la Información CMSI (2003). Declaración de principios construir la sociedad de la información: Un desafío global para el nuevo milenio (Informe). Ginebra: CMSI - Organización de las Naciones Unidas (ONU) - Unión Internacional de Telecomunicaciones (UIT).

Pérez, R. (1994). Investigación y mejora de programas. Programas comunes. En V.G. (Dir.), Problemas y métodos de la investigación en educación personalizada. (Págs. 510 - 536). Madrid, España: Madrid Rialp. 